\title{
Révision des directives «Collaboration corps médical-industrie»
}

\author{
Hermann Amstad ${ }^{a}$, \\ Walter Reinhart ${ }^{b}$ \\ a Dr, Secrétaire général \\ de l'ASSM, Bâle \\ b Prof. Dr, Président de la \\ «Commission consultative», \\ médecin-chef du service de \\ médecine interne, Kantons- \\ spital Graubünden, Coire
}

Lors de sa séance de printemps, le Sénat de l'Académie Suisse des Sciences Médicales (ASSM) a adopté la mise en consultation des directives révisées concernant la «Collaboration corps médical-industrie». Un nouveau remaniement a ainsi lieu, après la révision de 2005 et la transformation en directives des recommandations initiales datant de 2002. La cadence de ces révisions montre que la conscience du problème subit une mutation, tant auprès du public que du corps médical.

En 2007, l'ASSM a mis en place une «Commission consultative», chargée d'assister et d'accompagner l'application des directives «Collaboration corps médical-industrie». Comme il ressort des rapports annuels de la commission, les thèmes traités par cette dernière étaient constamment similaires et avaient de toute évidence été réglés de manière peu claire ou insuffisante jusqu'ici dans les directives; citons entre autres le monosponsoring, la mention «demande de crédits déposée» dans les programmes, le «Ghost Writing» ou encore la participation à des «Advisory Boards». En ce qui concerne ce dernier point, la commission consultative a organisé un workshop destiné aux experts en automne 2011; les participantes et participants étaient unanimes sur la nécessité d'agir dans ce domaine, incitant ainsi la commission à demander le remaniement des directives au comité de direction de l'ASSM. Celui-ci a donné l'ordre correspondant en janvier 2012.

La commission a renoncé à élaborer une nouvelle version des directives, dans la mesure où celles-ci ont fait leurs preuves sur le fond. Seul le chapitre III (consacré à l'activité des experts) est entièrement neuf. La commission a essayé de combler les lacunes évidentes et d'apporter des précisions dans les autres chapitres. Dans le détail, il s'agit notamment des modifications suivantes:

\section{Préambule}

- Définition des «conflits d'intérêts»

- Ajout concernant les «principes» pour la conduite à adopter en cas de conflits d'intérêts

Correspondance: Dr Hermann Amstad ASSM

Petersplatz 13

CH-4051 Bâle

mail[at]samw.ch

\section{Chapitre I: Recherche clinique}

- I.5: Ajouts relatifs au contenu contractuel

- I.7 (nouveau): Publication d'un travail scientifique: qui est l'auteur (et qui ne l'est pas)?
Chapitre II: Formation prégraduée, postgraduée et continue

- II.2: Financement des formations postgraduées par les cotisations des participants et l'institution organisatrice. Sponsoring exclusivement accordé par plusieurs entreprises indépendantes les unes des autres; la mention «crédits demandés» n'est pas autorisée.

- II.2: Les symposiums satellites ne sont pas reconnus comme formation continue.

- II.6: Les participants payent au moins un tiers des frais de déplacement et d'hébergement.

- II.8 (nouveau): Sponsoring de chaires

\section{Chapitre III (nouveau): Activité des experts}

- III.1: Collaboration au sein des «Advisory Boards»

- III.2: En principe, la prestation de conseil n'est fournie que sur la base d'un contrat.

- III.3: Montant approprié des honoraires

- III.4: Communication des conflits d'intérêts lors de l'élaboration des Guidelines

- III.5: Participation à des études d'observation ou à des enquêtes en ligne exclusivement dans le cas de problématiques scientifiques

- III.6: Divulgation des conflits d'intérêts au sein des commissions chargées de l'achat de produits thérapeutiques

\section{Chapitre IV: Acceptation de prestations en espèces ou en nature}

- IV.2 (nouveau): Les médecins gèrent les échantillons gratuits de manière correcte et appropriée.

\section{Annexe}

- Actualisation des prescriptions et des textes de loi

Le secrétariat général de l'ASSM a transmis les directives révisées à toutes les sociétés spécialisées, sociétés cantonales de médecine ainsi qu'aux autres acteurs du système de santé afin qu'ils prennent position; la consultation dure jusqu'au 31 août 2012. Bien entendu, les réactions individuelles sont également les bienvenues. La commission consultative examinera soigneusement les prises de position et en tiendra compte dans la mesure du possible. Il est prévu que le Sénat de l'ASSM adopte la version définitive des directives lors de sa séance du 29 novembre 2012. 